\title{
Multi-modality imaging of inflammation and ischemia for assessment of myocardial injury in Covid-19
}

\author{
Abbas Yousefi-Koma, MD, ${ }^{a}$ Farah Naghashzadeh, MD, ${ }^{b}$ Gemma A. Figtree, \\ MBBS, DPhil, ${ }^{\mathrm{C}}$ Sanjay Patel, MBBS, PhD, ${ }^{\mathrm{d}}$ and Keyvan Karimi Galougahi, MD, \\ $\mathrm{PhD}^{\mathrm{d}}$ \\ a Chronic Respiratory Diseases Research Center, National Research Institute of Tuberculosis and \\ Lung Diseases, Shahid Beheshti University of Medical Sciences, Tehran, Iran \\ b Lung Transplantation Research Center, National Research Institute of Tuberculosis and Lung \\ Diseases, Shahid Beheshti University of Medical Sciences, Tehran, Iran \\ c Royal North Shore Hospital, Sydney, Australia \\ d Department of Cardiology, Royal Prince Alfred Hospital, Sydney, NSW, Australia
}

Received Jun 7, 2020; accepted Jun 8, 2020

doi: $10.1007 / \mathrm{s} 12350-020-02233-x$

\section{INTRODUCTION}

Coronarvirus disease-2019 (Covid-19) is associated with cardiovascular manifestations including type 2 myocardial injury, acute coronary syndromes, and acute/fulminant myocarditis, contributing to an overall worse prognosis.

\section{CASE SUMMARY}

A 69-year-old woman with a history of hypertension presented with dyspnea and chest pain at the peak of Covid-19 pandemic. ECG revealed left-bundlebranch block (long-standing) (Figure 1) and chest pain quickly resolved with medical therapy. Polymerase chain reaction (PCR) assay was positive for Covid-19. Chest CT revealed bilateral ground-glass opacities consistent with Covid-19 (Figure 1). Peak troponin was $40 \mathrm{ng} / \mathrm{ml} \quad(<0.02 \mathrm{ng} / \mathrm{ml})$. Echocardiography revealed septal, anterior, and apical hypokinesis. Lopinavir-ritonavir, dual anti-platelet therapy, statin, losartan, and beta-blocker were started. She had an overall uncomplicated course of Covid-19. Due to complete resolution of pain and active Covid-19,

Reprint requests: Keyvan Karimi Galougahi, MD, PhD, Department of Cardiology, Royal Prince Alfred Hospital, 75 Missenden Road, Sydney, NSW 2050, Australia; keyvan.karimi@hri.org.au J Nucl Cardiol 2021;28:3100-3.

$1071-3581 / \$ 34.00$

Copyright $\odot 2020$ American Society of Nuclear Cardiology angiography was deferred for when she was deemed non-infective.

To assess for myocarditis versus myocardial infarction/injury, on admission day $7,{ }^{18}$ FDG-PET with 18 -h fasting protocol was performed showing avid uptake in septum, anterior wall, and apex (Figure 2). A subsequent ${ }^{99 \mathrm{~m}} \mathrm{Tc}-\mathrm{MIBI}-\mathrm{SPECT}$ revealed rest perfusion defects in the same segments (Figure 2) (stress imaging not performed due to possible infarct).

While segmental FDG uptake (due to inflammation) with matching perfusion defects (due to inflammatory microvascular dysfunction) are typical for myocarditis, ${ }^{1}$ in this context secondary to Covid-19 shown to cause myocarditis and cardiac microvascular involvement ${ }^{2}$; acute inflammatory response to myocardial infarction precipitated by Covid-19 may generate a similar pattern. ${ }^{3}$ This perfusion-metabolism mismatch should not be misinterpreted with myocardial uptake indicating myocardial viability in an ischemic left anterior descending (LAD) artery territory since the prolonged fasting protocol suppresses myocardial glucose uptake. ${ }^{3}$

Angiography performed when the patient became PCR negative with resolution of pulmonary involvement revealed severe LAD artery disease (Figure 3), confirming anterior wall infarction precipitated by Covid-19 that was treated with percutaneous coronary intervention. 

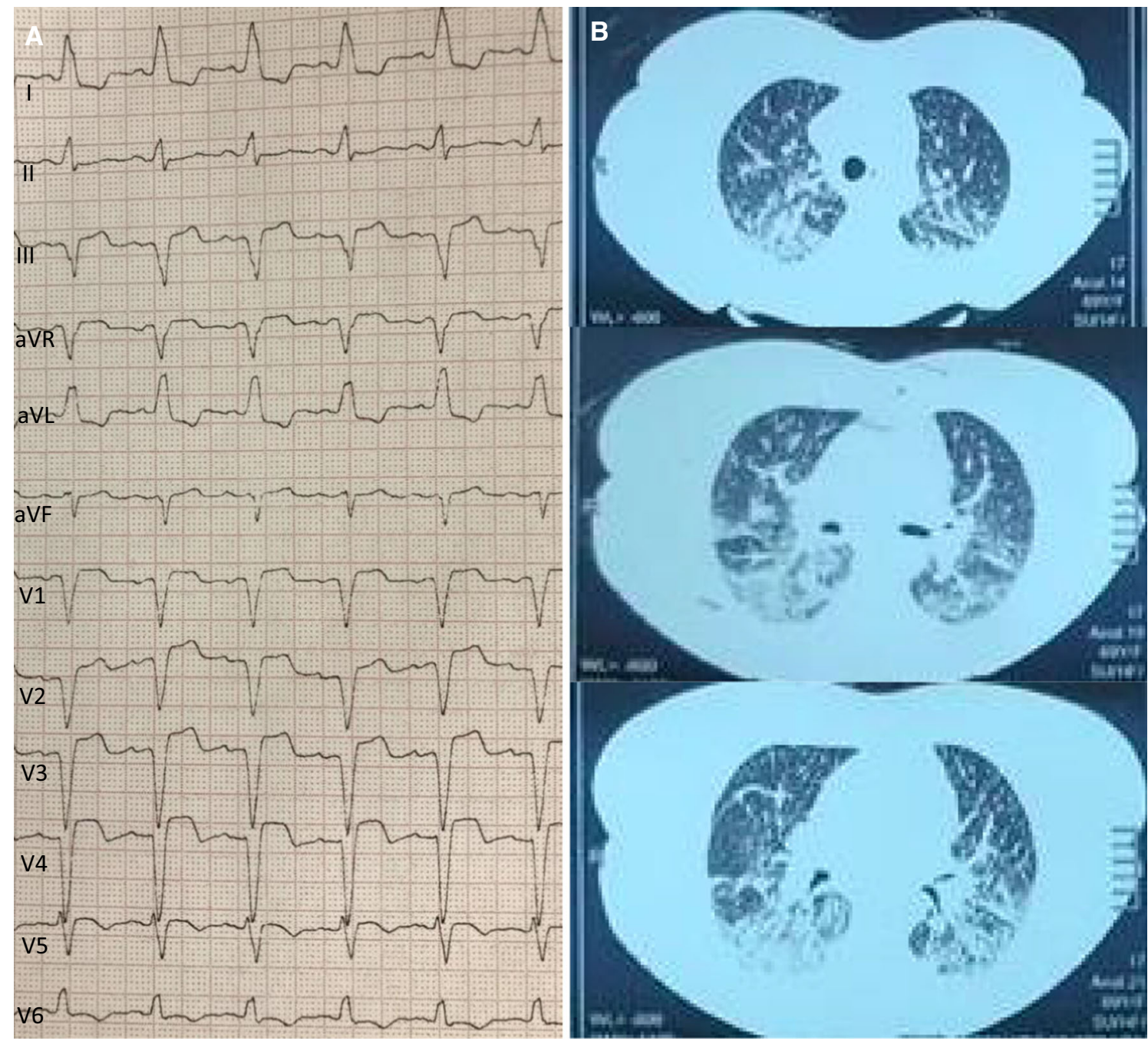

Figure 1. Initial investigations. A ECG revealed left-bundle-branch block pattern (old) with anteroseptal Q waves. B Chest computed tomography revealed multilobar ground-glass opacities consistent with Covid-19. 
A

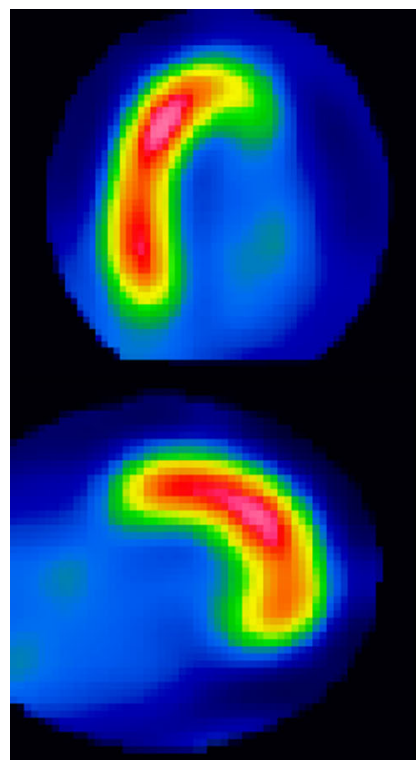

SPECT-Perfusion

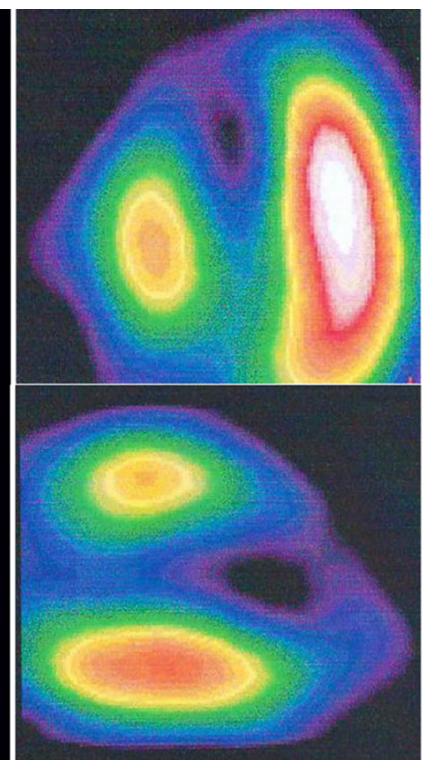

B

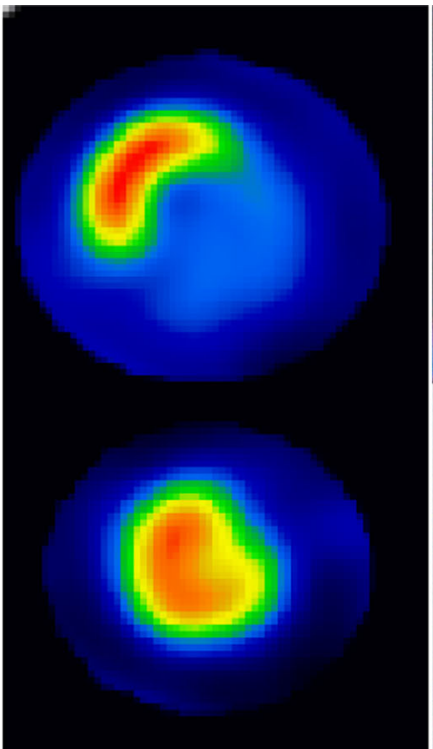

SPECT-Perfusion

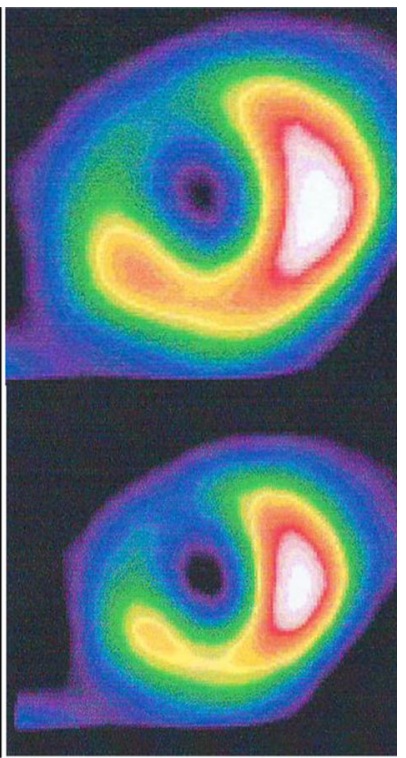

Figure 2. Cardiac ${ }^{18}$ FDG-PET and ${ }^{99 m}$ Tc-MIBI-SPECT perfusion imaging. Horizontal long-axis, vertical and short-axis slices show avid FDG uptake in the septum, anterior wall, anteroseptal, and apical segments and suppression of FDG uptake in the rest of myocardium, with matching segmental defects on the rest SPECT perfusion images.
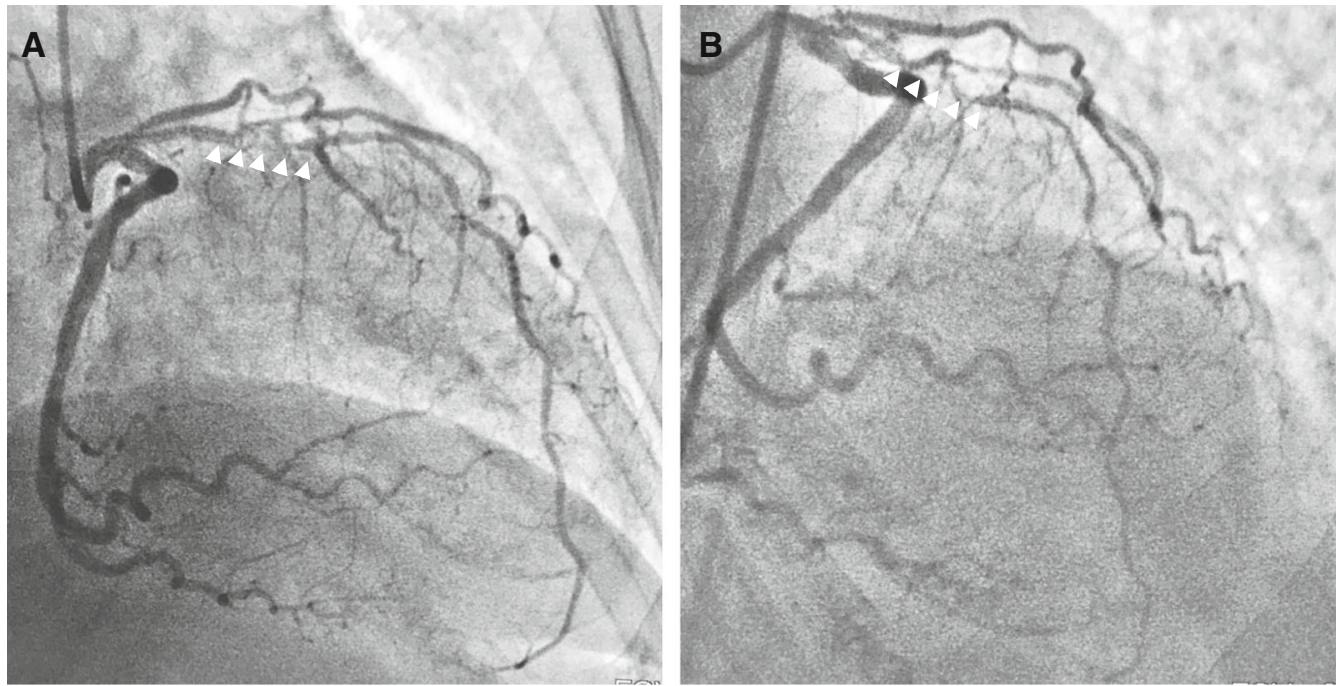

Figure 3. Coronary angiography. Severe stenosis in the mid-segment of the left anterior descending artery (arrowheads) in bifurcation with a diagonal branch in the right anterior oblique and anterior-posterior cranial projections. 


\section{Disclosures}

Sanjay Patel: Educational grants-Abbott Vascular and Novartis, consultancy fees-Abbott Vascular, Novartis, and Aspen. Gemma Figtree: Consultancy fees-Janssen, CSL. Other authors declare no conflict of interest.

\section{References}

1. Niederkohr RD, Daniels C, Raman SV. Concordant findings on myocardial perfusion SPECT and cardiac magnetic resonance imaging in a patient with myocarditis. J Nucl Cardiol 2008;15:4668.

2. Fox SE, Akmatbekov A, Harbert JL et al. Pulmonary and Cardiac Pathology in Covid-19: The First Autopsy Series from New Orleans. medRxiv; Accessed at https://www.medrxiv.org/content/ 10.1101/2020.04.06.20050575v1.full.pdf.

3. Manabe O, Oyama-Manabe N, Naya M et al. Pitfalls of (18)F-FDG PET for evaluating myocardial viability. $J$ Nucl Cardiol 2017;24:1110-3.

Publisher's Note Springer Nature remains neutral with regard to jurisdictional claims in published maps and institutional affiliations. 